Revista General de Información y Documentación ISSN: 1132-1873

http://dx.doi.org/10.5209/rgid.72817

\title{
Coautoría y revisión por pares: prácticas y percepciones de los investigadores noveles españoles
}

\author{
Blanca Rodríguez-Bravoㅜㄹ David Nicholas ${ }^{2}$
}

Recibido: 26 de junio de 2020 / Aceptado: 7 de octubre de 2020

Resumen. El estudio analiza las opiniones y prácticas de los investigadores españoles noveles (Early Career Researchers o ECRs) respecto a la coautoría y a la revisión por pares según datos obtenidos de una encuesta. Se observa que los ECRs contribuyen a todas las tareas de una investigación aunque no siempre se les reconozca suficientemente el mérito. Desearían que el orden de firma estuviera regulado y reflejara el trabajo de cada autor. Asimismo, participan activamente en el proceso de arbitraje tanto en el papel de autores como en el de revisores. Abogan por mejoras en la revisión por pares pero se manifiestan medianamente satisfechos con un proceso que, aunque duro, resulta enriquecedor. Respecto a las alternativas son conservadores, prefieren estar protegidos por el anonimato y, consecuentemente, apoyan el modelo de doble ciego.

Palabras clave: Coautoría; Comunicación académica; Encuestas; España; Investigadores noveles; Revisión por pares.

\section{[en] Authorship and Peer Review: Practices and Perception of Spanish Early Career Researchers}

\begin{abstract}
The study analyzes the pinions and practices of the Spanish Early Career Researchers (ECRs) regarding co-authorship and peer review obtained from a survey's data. It is observed that ECRs contribute to all the tasks of an investigation, although their merit is not always sufficiently recognized. They would like the signing order to be regulated and reflect the work of each author. They also actively participate in the peer review process, both in the role of authors and of reviewers. They ask for improvements in reviewing but they seem moderately satisfied with a process that, although hard, is enriching. Between the possibilities they are conservative, prefer to be protected by anonymity and consequently support the double-blind model.
\end{abstract}

Keywords: Authorship; Early career researchers; Peer review; Scholarly communication; Spain; Survey.

Sumario. 1. Introducción. 2. Contexto, Métodos y Objetivos. 3. Resultados y discusión. 4. Discusión y conclusiones. 5. Referencias bibliográficas.

$1 \quad$ Universidad de León. Biblioteconomía y Documentación

E-mail: blanca.rodriguez@unileon.es

2 Ciber Research Ltd.

E-mail: Dave.Nicholas@ciber-research.eu 
Cómo citar: Rodríguez-Bravo, B.; Nicholas, D. (2020) Coautoría y revisión por pares: prácticas y percepciones de los investigadores noveles españoles, en Revista General de Información y Documentación 30 (2), 379-399.

\section{Introducción}

La autoría y el arbitraje son dos caras de la misma moneda. De un lado la producción de contenidos y de otro el control de calidad de lo que se produce y ambos procesos están a cargo de los mismos científicos (Nicholas et al., 2015). En este estudio se aborda la experiencia del colectivo de jóvenes investigadores o Early Career Researchers (ECRs), aquellos investigadores que están realizando el doctorado o bien son doctores pero que no han alcanzado una posición de estabilidad laboral. Indagar sobre este sector tiene sentido porque constituyen el presente y el futuro de la ciencia y, como se verá en esta investigación, son su principal fuerza de trabajo. Son autores, son revisores y participan en todos los procesos que conforman una investigación (Nicholas et al., 2019).

Como han puesto de relieve Rodríguez-Bravo y Nicholas (2019) en un trabajo derivado del Proyecto Harbingers ${ }^{3}$ que se condujo por medio de entrevistas a investigadores noveles realizadas entre 2016 y 2018, los ECRs en general y los españoles en particular están focalizados en publicar. Persiguen la publicación en revistas selectas y en las principales posiciones, porque son las que creen pueden ayudarles a conseguir situarse en la academia de forma permanente. Cuanto más tiempo llevan en la ciencia más sienten la presión de publicar en revistas prestigiosas debido a que están más al tanto de las políticas de evaluación a las que se les va a someter y son conscientes de que se encuentran en un sector muy competitivo (Rodríguez-Bravo y Nicholas, 2019).

El trabajo de Nicholas et al. (2017) basado en las mencionadas entrevistas a un centenar de ECRs pone de relieve que los investigadores jóvenes son generalmente autores productivos, algunos incluso prolíficos, comprometidos con la participación en todas las fases de la investigación. En teoría el orden de firma de los artículos se deriva de la contribución al trabajo realizado pero no siempre es así y esta situación, que se percibe injusta, crea malestar. Los ECRs abogan por que se fijen claramente los criterios para establecer el orden de firma. La problemática de la fijación de criterios de autoría ha cobrado relevancia debido al crecimiento de la colaboración de las últimas décadas que ha conducido a que los autores de los textos se hayan incrementado considerablemente. La secuencia de los autores es la única pista que permite otorgar crédito a los firmantes. Existen muchas diferencias entre disciplinas en esta cuestión. Hay sectores donde la norma es el orden alfabético como ha constatado Weber (2018) quien indica que en algunas ramas como las matemáticas, la física y algunas ciencias sociales más del $60 \%$ de los artículos utilizan el orden alfabético. No obstante, esta no es la situación dominante en el entorno de los ECRs

3 Se trata del proyecto: Early Career Researchers: The Harbingers of Change? (Proyecto Harbingers) Financiado por Publishing Research Consortium (2016-2018). Más información en: http://ciber-research.eu/harbingers.html 
entrevistados por Nicholas et al. (2017) y, así, estos investigadores aspiraban a las posiciones que consideraban relevantes, primer autor y autor de correspondencia. En el caso español frecuentemente figuraban como primer autor porque gran parte de los resultados que publican proceden de sus tesis doctorales. Con frecuencia sus mentores son autores de correspondencia y se sitúan en la última posición, como han indicado para el caso español Rodríguez-Bravo y Nicholas (2019).

Los mismos autores apuntan que en el primer año de entrevistas (2016) los investigadores noveles defendían con pasión que el orden de autoría fuera estrictamente el merecido por el trabajo invertido en la investigación y/o en la escritura del artículo. En los años posteriores, 2017 y 2018, algunos investigadores se muestran más pragmáticos y, como señalaba una de las investigadoras, "ayudamos a quien necesita una promoción dejándole figurar como primer autor”. Otra investigadora de ciencias de la salud apuntaba: “este año soy primera autora en todos los artículos para obtener la Miguel Servet, después pasaré a última posición y a autor de correspondencia". Otro investigador se manifestaba así: "Trato de publicar mucho y lo más alto posible como senior (último autor o autor de correspondencia)”. Los investigadores españoles afirmaban que quienes trabajaban en grupos grandes y con una trayectoria consolidada obtenían ventaja competitiva pues colaboraban más, publicaban más y podían hacerlo en acceso abierto obteniendo más citas. La contrapartida es, apuntaban, el incremento del número de posibles firmantes. La colaboración entre grupos y laboratorios ha conducido, de hecho, a la práctica de algunas revistas de facilitar la posibilidad de compartir estas posiciones: co-primer autor y co-autor de correspondencia.

En la senda de la publicación se encuentra el escollo de la revisión por pares; superar el filtro del arbitraje es crucial. La revisión por pares de los manuscritos enviados a revistas científicas es considerada esencial para mantener la integridad de la literatura científica (Baldwin, 2018; Tennant, 2017). Señalan Merga, Mason y Morris (2018) que el proceso de publicación y de revisión es complejo para los jóvenes investigadores y estos aprenden su funcionamiento participando en él. De hecho, los investigadores noveles contribuyen con frecuencia al proceso. Así, en una encuesta reciente realizada en el ámbito de las ciencias de la vida, el 92\% de las respuestas indica que estos investigadores han participado en la revisión de al menos un manuscrito (Inside eLife, 2018). Asimismo, más de la mitad de los ECRs que contribuyeron al cuestionario afirmaron haber revisado un manuscrito sin ayuda de su mentor. Como pone de relieve McDowell (2018) los editores de las revistas quizá no sean totalmente conscientes de hasta qué punto los investigadores noveles participan en este proceso, de hecho Patterson y Schekman (2018) afirman que es una práctica común en los investigadores que lideran grupos la petición de ayuda a otros investigadores postdoctorales o predoctorales experimentados para llevar a cabo el proceso de peer review, si bien esta contribución no siempre es reconocida. Un estudio reciente de McDowell et al. (2019) explora, por medio de una encuesta a ECRs su participación en el proceso como co-revisores, con conocimiento de su participación por la revista o como revisores fantasma (ghostwriting), cuando la revista desconoce esa aportación y asigna la revisión al investigador senior. 
Señalan Rodríguez-Bravo et al. (2017) y Rodríguez-Bravo y Nicholas (2019) que los jóvenes investigadores tienen generalmente experiencia en el proceso de arbitraje, tanto respondiendo a los revisores como actuando en esa posición ellos mismos. Con base en las entrevistas del proyecto mencionado, Nicholas et al., (2019) apunta que dado que mayoritariamente trabajan en grupos en muchos casos contestan a los revisores por delegación de sus mentores. Se muestran relativamente positivos con el sistema de arbitraje pero cautos en sus preferencias. Se inclinan por el sistema de doble ciego que permite que estos investigadores actúen con libertad como evaluadores y evite que sean perjudicados en las evaluaciones de sus manuscritos. Como autores se quejan de que las revisiones tardan en llegar y como revisores que no se les concede suficiente tiempo para realizar un buen trabajo (Rodríguez-Bravo et al., 2017). Todos en general y los españoles en particular consideran necesaria una mejor selección de los revisores, formación para acometer la tarea, instrucciones claras de las revistas y reconocimiento por la tarea desempeñada (Rodríguez-Bravo y Nicholas, 2019).

\section{Contexto, Métodos y Objetivos}

Este artículo se propone presentar algunos resultados de la segunda fase del Proyecto Harbingers cuyo nombre completo es "Early Career Researchers: the Harbingers of Change?”. Ha sido liderado por David Nicholas, CIBER Research, Ltd. y subvencionado por Publishing Research Consortium.

El Proyecto Harbingers se inició en 2016 y persige determinar si la nueva ola de investigadores está contribuyendo a cambiar el escenario de la comunicación académica. Se pretende conocer si su comportamiento se diferencia del de sus mentores o, por el contrario, continúan los patrones tradicionales. A efectos de este proyecto se entiende por ECRs aquellos investigadores que están realizando el doctorado o bien son doctores pero que no han alcanzado una posición de estabilidad.

En una primera fase, el estudio longitudinal consistió en la realización de un centenar de entrevistas que se repitieron durante tres años (2016-2018) a investigadores en los inicios de su carrera donde dos terceras partes procedían de ámbitos científicos y una tercera parte de ciencias sociales. Los Early Career Researchers procedían de 7 países -China, España, Francia, Malasia, Polonia, Reino Unido y USA-. Los instrumentos de recolección, informes y publicaciones derivadas se relacionan en la página de Ciber Research Ltd.(http://ciberresearch.eu/CIBER_projects.html).

Los datos recabados de las entrevistas y el análisis realizado de ellos resultaron esenciales para el diseño del cuestionario ${ }^{4}$ elaborado para la segunda fase del proyecto a la que se alude aquí. Así, el cuestionario se desarrolló con base en el guion de las entrevistas y la experiencia obtenida durante la investigación previa fue esencial para acometer el presente estudio. Se sabía ya qué preguntar y cómo hacerlo. Para que el cuestionario no resultase tedioso no se recogieron en él todas las

4 http://ciber-research.eu/download/ECR-questionnaire-for-website-20191129.pdf 
cuestiones formuladas durante las entrevistas si no aquellas consideradas estratégicas o que requerían confirmación. El propósito de esta segunda fase era extender el estudio a una población mayor y más diversa de ECRs que incluyera a los científicos de humanidades.

El cuestionario se diseñó en inglés, pero se tradujo a las lenguas nacionales de los investigadores participantes en el proyecto mencionado para ampliar su radio de alcance y potenciar la respuesta. Entre estas lenguas se encontraba el español. Se testaron todas las versiones del cuestionario antes de hospedarlo en SurveyMonkey y se abrió el 10 de mayo de 2019 cerrándose la recogida de respuestas el 30 de julio de 2019.

No fue posible establecer un único medio de diseminación del enlace al cuestionario al no existir un directorio de ECRs. En el caso del enlace al cuestionario traducido al español, este se distribuyó a través listas de usuarios de universidades, por medio de listas de correo del ámbito científico del investigador a cargo, gracias a la mediación de editores y a través de redes sociales académicas (ResearchGate). Asimismo, colaboraron en su distribución algunas asociaciones de jóvenes investigadores constituidas en universidades por medio de sus redes de miembros.

El cuestionario se iniciaba con una cuestión binaria (sí/no) preguntando a quienes accedían si eran o no ECRs. Si contestaban negativamente su aportación se eliminaba para el análisis realizado. En el caso que nos ocupa, el cuestionario distribuido en español, se obtuvieron 158 respuestas que una vez filtradas y eliminados los 21 investigadores que contestaron no ser ECRs se limitaron a 137 respuestas que se procedió a analizar. Las preguntas demográficas se situaban al final del cuestionario. Hay que considerar que los encuestados eran libres de contribuir a las preguntas que consideraban oportunas y, por tanto, ninguna pregunta obtiene 137 respuestas.

Existen diferentes tipos de preguntas, algunas dicotómicas, otras en las que se pedía elegir una o más respuestas. En otras se ofrecían opciones siguiendo una escala de Likert. Las opciones de las preguntas en la escala de Likert usadas en el cuestionario son: "Mucho, Bastante, Algo/Poco, Muy poco, Nada, No sé”. Los valores medios de estas preguntas se calcularon en base a los valores numéricos de los ítems con "Nada" teniendo un valor de 1 y "Mucho" con un valor de 5. Una media de 3 se consideró indicativa de que una alternativa recibe una aquiescencia intermedia, de "poco" que viene a ser equivalente a "algo". Una media de 4 refleja un interés notable por una práctica y un peso de 2 reflejaría un escaso interés en el ítem por parte de los investigadores que han cubierto el cuestionario.

En este artículo no se pretende analizar todos los datos obtenidos. Por el contrario, se centrará en las cuestiones que perseguían profundizar en el papel desempeñado por los investigadores noveles españoles como autores y como revisores Este estudio se dedicará a analizar de manera única y específica las respuestas al cuestionario traducido al español y que recogió información de investigadores españoles.

En el presente estudio se busca indagar en:

1. La participación de ECRs en el conjunto de las actividades que conforman la autoría.

2. La existencia de políticas de autoría y la influencia de los ECRs en las decisiones sobre la autoría. 
3. La participación de los ECRs en el proceso de peer review como autores y su opinión sobre el proceso.

4. La participación de los ECRs en el proceso de peer review como revisores, los beneficios conseguidos y su opinión sobre el proceso.

5. La valoración, preferencias y sugerencias de los ECRs sobre los modelos de revisión por pares.

Se presentan a continuación los resultados obtenidos de las cuestiones demográficas, así como de las que persiguen obtener información sobre los aspectos recogidos en los objetivos.

\section{Resultados y discusión}

Respecto a la información recogida de la encuesta distribuida en español, se trata de un colectivo constituido principalmente por investigadores que trabajan en España (75,3\%), como era previsible. Con todo, es significativo el porcentaje de investigadores asentados en diversos países de tres continentes. Estos datos permiten observar que no son pocos aquellos investigadores que han salido de España en busca de una continuidad en su carrera investigadora, situación observada por RodríguezBravo y Nicholas (2019). Respecto a su género, existe una práctica paridad con $52,7 \%$ de mujeres y 46,1\% de hombres (hay un mínimo porcentaje de ECRs que no responde a la pregunta). La franja de edad predominante es la señalada por la horquilla de los 26-30 años (34,8\%), seguida por aquella de entre 31 y 35 (27\%). Aquí interesa subrayar el hecho de que han participado en la encuesta un no desdeñable número de investigadores de 36 o más años $(28,1 \%)$ que se consideran a sí mismos ECRs y ello no debido a su falta de experiencia, sino más bien a la ausencia de posiciones estables en la investigación, situación con una incidencia superior en España que en otros países (Rodríguez-Bravo; Nicholas, 2019).

En relación a su formación académica, más de la mitad de los participantes tienen una formación de Máster (58,2\%) y se definan como estudiantes de doctorado (59,3\%). Complementariamente, el 31,9\% de los investigadores manifiestan ser ya doctores. Preguntados sobre el número de años que llevan en la investigación, se confirma la escasez de posiciones estables en la misma durante las últimas dos décadas. De hecho, un tercio de los investigadores que respondieron llevan 6 años o más como científicos no estables e incluso algunos (9,9\%) superan la decena de años. Respecto a las áreas científicas en que se inscriben, predominan los científicos de ciencias sociales (52,7\%), seguidos de los de ciencias de la vida (17,6\%), artes y humanidades (16,5\%), ciencias puras e ingeniería $(8,6 \%)$ y ciencias de la salud $(4,4 \%)$. En el cómputo global de respuestas al cuestionario, es decir los resultados obtenidos en el conjunto de los países donde se distribuyó la encuesta (Nicholas et al., 2020b), se confirma la considerable participación de investigadores de ciencias sociales si bien el sesgo hacia estas materias es inferior al español limitándose el porcentaje a un tercio del total (33,9\%) y con una contribución superior de científicos de ciencias puras e ingenierías (20,7\%) y de ciencias de la salud (9,7\%). La 
participación de investigadores de ciencias de la vida es equiparable (16,3\%) y la de artes y humanidades es prácticamente la mitad (8,6\%).

Dividiremos los resultados referidos a la autoría y a la revisión por pares según los objetivos planteados en el apartado anterior.

\subsection{La participación de los ECRs en el conjunto de actividades que conforman la autoría}

Se inicia este apartado del cuestionario preguntando a los participantes si habían publicado algún artículo en coautoría. De 116 personas que respondieron, el 75,8\% lo hicieron afirmativamente. El porcentaje obtenido es relativamente alto, máxime si consideramos la elevada participación en el cuestionario de investigadores de ciencias sociales y de artes y humanidades que tienen una mayor tendencia a trabajar en solitario.

Las siguientes cuestiones del apartado dedicado a la coautoría se dirigieron solamente a quienes habían publicado en colaboración. Las contribuciones en un artículo pueden ser de distinto tenor. Los participantes podían señalar tantas respuestas como fueran necesarias entre las que aparecen listadas en la Tabla I.

Tabla I. ¿Cuál ha sido tu contribución en los artículos en los que has participado como coautor?

\begin{tabular}{|l|l|l|}
\hline Opciones & $\mathbf{N . 8 5}$ & $\mathbf{\%}$ \\
\hline Escritura del artículo & 75 & 88,2 \\
\hline Análisis de los datos & 69 & 81,2 \\
\hline Revisión de la literatura & 64 & 75,3 \\
\hline Realización del trabajo de campo & 63 & 74,1 \\
\hline Producción o recopilación de datos & 63 & 74,1 \\
\hline Edición del artículo & 51 & 60,0 \\
\hline Conseguir fondos para la investigación & 4 & 4,7 \\
\hline $\begin{array}{l}\text { Conseguir fondos para publicar en acceso } \\
\text { abierto (APCs) }\end{array}$ & 1 & 1,2 \\
\hline
\end{tabular}

Como puede observarse en la Tabla I, los ECR españoles participan en todas las tareas implicadas en la producción de un artículo. La escritura del manuscrito y el análisis de datos son las dos actividades más usuales. Los investigadores noveles también se encuentran notablemente involucrados en la revisión de la literatura, en la realización del trabajo de campo y en la producción o recopilación de datos.

Por el contrario, la consecución de financiación no es relevante en el global de las respuestas y todavía más marginal es la búsqueda de fondos para publicar en abierto. Se deduce que la publicación en abierto, es una opción a valorar solo si se dispone de fondos para la investigación. Así lo han constatado previamente Rodríguez y Nicholas (2019). Como es sabido, la obtención de fondos para la investigación de diversas convocatorias ya obliga a los científicos españoles a publicar en open access. 
Tres investigadores señalaron otras razones. Dos de ellos hacían referencia, con otras palabras, a las opciones $4^{\mathrm{a}}$ y/o $5^{\mathrm{a}}$ de la Tabla I: "Contribución en resultados técnicos” y "Realización de los experimentos”. La tercera respuesta reforzaría las principales opciones señaladas: "He hecho todo, el resto ha firmado. No he conseguido fondos porque no existen y en la mayoría de los casos los fondos provienen de mi bolsillo". Esta última opinión pone de relieve la situación de bastantes investigadores españoles y su malestar con los criterios de inclusión de autores en la firma de los artículos.

La pregunta que responde a las opciones planteadas en la tabla I permite forjar la imagen de unos investigadores noveles multitarea y muy involucrados en la investigación, pero interesa, asimismo, conocer hasta qué punto tienen influencia en las decisiones que se toman sobre el artículo cuando son coautores. De 87 respuestas que obtenidas, el 44,8\% afirma tener una gran influencia, el 48,3\% tener solo cierta influencia y únicamente el 6,9\% contesta no tener influencia alguna. Así, en un 93,1\% de los casos los encuestados se consideran influyentes en el proceso de autoría de un artículo en colaboración. Como han constatado previamente Nicholas et al. (2017) y Rodríguez-Bravo y Nicholas (2019) los investigadores noveles con frecuencia imponen sus opciones también en la selección del outlet donde difundir artículos en colaboración. En el último trabajo citado, los autores indicaron que los ECRs españoles no estaban satisfechos con las decisiones que se tomaban acerca de quién firmaba los artículos ni en qué orden se firmaba. Se deducía que los criterios no estaban en general claramente establecidos.

\subsection{La existencia de políticas de autoría y la influencia de los ECRs en las decisiones sobre la autoría}

La circunstancia señalada en el párrafo anterior se confirma en este estudio dado que poco más de una tercera parte de quienes responden al cuestionario afirma que exista una política de autoría en su grupo de investigación que determine el estatus de autoría y el orden (38,8\%). El 31,9 \% respondieron que no existía, y el 29,3\% no sabía si existía o no. Esta pregunta fue respondida por un buen número de participantes que no habían publicado en coautoría, dado el elevado número de respuestas (N. 116). De ahí, tal vez, el alto número de investigadores que se ha inclinado por contestar "no sé". Como se deduce de otros estudios (Jamali et al., 2020), las circunstancias a este respecto varían mucho entre países. La limitada respuesta afirmativa en España acerca de la existencia de políticas al respecto se aproxima a la del Reino Unido donde el porcentaje es incluso inferior (32,1\%) y se aleja de China $(91,5 \%)$ y de Rusia $(72,1 \%)$ donde aparentemente la autoría está mucho más normativizada. La ausencia de políticas en este sentido en España y en Reino Unido ya fue confirmada previamente en otro trabajo vinculado al Proyecto Harbingers (Nicholas et al., 2017).

A continuación, se solicitaba conocer a qué aspectos afectaba esa política de autoría y se pedía a los investigadores que señalasen las respuestas apropiadas entre las cuatro mostradas o que especificasen otros criterios. En esta ocasión la respuesta se reduce a quienes habían contestado afirmativamente a la pregunta anterior. 
Tabla II. ¿Cuáles son las principales características de esta política de autoría?

\begin{tabular}{|l|l|l|}
\hline Opciones & N. 42 & \% \\
\hline $\begin{array}{l}\text { Criterios para determinar quién puede ser incluido como } \\
\text { autor del artículo }\end{array}$ & 32 & 76,2 \\
\hline $\begin{array}{l}\text { Criterios para determinar el orden de posición de los } \\
\text { autores }\end{array}$ & 32 & 76,2 \\
\hline $\begin{array}{l}\text { Criterios para determinar quién es el autor de } \\
\text { correspondencia }\end{array}$ & 11 & 26,2 \\
\hline Criterios para determinar quién es el primer autor & 32 & 76,2 \\
\hline
\end{tabular}

Se observa que la política de autoría que siguen los grupos de investigación guarda una estrecha relación con la asignación de autores, el orden de firma y los criterios de determinación del primer autor. A la asignación del autor de correspondencia parece que no se le presta tanta atención y está menos regulada que en otros países donde el cuestionario ha recabado información. En el conjunto de los países donde el cuestionario ha obtenido respuestas, la determinación del primer autor y el orden de posición son los criterios considerados principales (Jamali et al., 2020).

En la siguiente pregunta se cuestionaba a los investigadores noveles si actuarían de distinta manera si pudieran tomar las decisiones. Se constata que la insatisfacción con las reglas vigentes (o con la ausencia de regulación) es alta, ya que un 44,2\% de los 43 investigadores que respondieron lo hicieron afirmativamente, el 32,6\% contestaron negativamente y un 23,3\% dijeron que no sabían. En las entrevistas realizadas a ECRs españoles entre 2016 y 2018 ya se puso de manifiesto una situación semejante (Rodríguez-Bravo y Nicholas, 2019).

A quienes afirmaron que modificarían las prácticas si estuviese en su mano se les pregunta sobre qué cambiarían. Solo 13 de los 19 investigadores proclives al cambio respondieron:

Tabla III ¿Qué cambiarías?

\begin{tabular}{|l|l|}
\hline 1 & $\begin{array}{l}\text { "Cuando hay jerarquías se imponen ciertos aspectos en la firma de artículos. Mientras } \\
\text { más diferencias hay en la jerarquía más problemas hay. También se complica todo } \\
\text { cuando es un proyecto de investigación donde hay muchos investigadores. El método } \\
\text { ideal sería firmar en función del trabajo hecho no de la jerarquía. Fin.” }\end{array}$ \\
\hline 2 & $\begin{array}{l}\text { "Probablemente el orden en el cual aparecen los autores. Siento que a veces (debido a } \\
\text { que la mayoría de mis publicaciones son en inglés o francés) mis coautores nativos } \\
\text { de esas lenguas se dedican más a corregir ortografía y gramática que a aportar ideas } \\
\text { para el texto. Echo en falta una discusión sustancial más abundante y frecuente.” }\end{array}$ \\
\hline 3 & "Que el orden de la autoría no contara tanto y que se pudiera publicar en abierto.” \\
\hline 4 & "El porcentaje y tipo concreto de autoría.” \\
\hline 5 & $\begin{array}{l}\text { "Indicaría exactamente qué ha hecho cada coautor con un porcentaje que pudiese } \\
\text { fijar más justamente la contribución individual.” }\end{array}$ \\
\hline 6 & "La comunicación dentro del grupo es muy importante.” \\
\hline 7 & $\begin{array}{l}\text { "Que los criterios de participación fueran fijos, no que solo se apliquen cuando } \\
\text { convenga.” }\end{array}$ \\
\hline
\end{tabular}




\begin{tabular}{|l|l|}
\hline 8 & $\begin{array}{l}\text { "No me parece correcto que se sitúe en primera posición a quien ha concebido la } \\
\text { investigación, y a quienes han realizado el trabajo (predoctorales) detrás. La jerarquía } \\
\text { no debiese aplicarse." }\end{array}$ \\
\hline 9 & "El IP del grupo no siempre es el verdadero corresponding author.” \\
\hline 10 & $\begin{array}{l}\text { "Publicaría mucho más pues podría invitar a otros investigadores internacionales a } \\
\text { compartir mi investigación ya que abordan en la suya otros aspectos del mismo } \\
\text { tema.” }\end{array}$ \\
\hline 11 & "Criterios más estrictos para considerar coautorías.” \\
\hline 12 & $\begin{array}{l}\text { "Aspectos del contenido que a veces se quieren contemplar pero el coautor considera } \\
\text { innecesarios.” }\end{array}$ \\
\hline 13 & "Liderazgo cooperativo y mayor comunicación.” \\
\hline
\end{tabular}

El descontento que manifiestan guarda relación con las variables consideradas más arriba (Tabla II): asignación de autorías inmerecidas; orden de autores que no refleja el trabajo llevado a cabo; que los seniors ocupen las posiciones principales sin haber realizado una contribución que lo justifique. Asimismo, agradecerían que existiese una política estable que fijase el orden utilizando siempre los mismos criterios y, en su defecto, echan de menos un mayor diálogo en el grupo para fijar el orden.

En suma, los ECRs se sienten injustamente tratados porque su trabajo no se refleja en la firma de los artículos. Su preocupación obedece a que, en el entorno competitivo en que se mueven y en la rigurosa evaluación a que están sometidos, para poder seguir en un trabajo científico se valora, principalmente, figurar como primeros autores o como autores de correspondencia. En 2019, Rodríguez-Bravo y Nicholas apuntaban la circunstancia de una investigadora que era crítica respecto a ampliar la colaboración con otros grupos, pese a sus constatadas ventajas, porque con ello se alejaban las posibilidades de ocupar posiciones relevantes de firma.

\subsection{La participación de los ECRs en el proceso de peer review como autores y su opinión sobre el proceso}

Las cuestiones relativas a las prácticas de peer review tienen una doble vertiente. De un lado, se indaga en si los ECRs han asumido la responsabilidad de contestar a los revisores cuando han recibido la evaluación de artículos de los que han sido coautores. De otro, se trata de conocer si ellos han actuado como revisores del trabajo de otros autores y su experiencia al respecto.

Se comienza preguntando si tienen experiencia respondiendo a los revisores acerca de sus manuscritos, bien elaborados en investigaciones individuales o bien en colaboración. De 111 respuestas, el 63,9\% respondió afirmativamente y el 36\% lo hizo negativamente. Se constata que una mayoría de los investigadores encuestados tienen experiencia respondiendo a las consideraciones de los revisores, si bien el porcentaje en España es inferior al que aporta en su estudio Jamali et al. (2020) con referencia a los datos del cuestionario en el conjunto de los países donde el cuestionario ha recabado información. Esta situación reflejaría que en otros contextos los ECRs asumen una mayor responsabilidad en esta fase del proceso de publicación, independientemente o como co-revisores (Patterson y Schekman, 2018; McDowell et al., 2019). 
A quienes contestaron afirmativamente se les pide que valoren su experiencia. De 71 respuestas el 43,7\% de los participantes valoraron la experiencia como buena, un porcentaje superior $(52,1 \%)$ la consideró variable dependiendo de la ocasión y solo tres encuestados $(4,2 \%)$ la calificaron de mala. La experiencia de los jóvenes investigadores respondiendo a los revisores es, por tanto, irregular. Esta circunstancia ha sido constatada previamente en el trabajo de Rodríguez-Bravo et al., (2017) donde una investigadora señalaba que cada revisión era “una lotería”. El mismo estudio indicaba que los españoles se mostraban menos positivos que en otros seis países, solo el 29\% calificaba la experiencia de buena. Pese a ello la consideraban enriquecedora.

Con todo, en el cuestionario analizado se aprecia que los ECRs tienen una aproximación relativamente positiva al proceso de peer review dado que son limitados quienes califican la experiencia de mala. El trabajo de Jamali, et al. (2020) apunta, sin embargo, que los españoles son algo menos positivos que el global de ECRs encuestados. En esta cuestión se han observado grandes disparidades entre los países. Los norteamericanos son los más negativos y los rusos seguidos de los chinos los más positivos. Nuevamente los datos de los españoles se asemejan a los de los británicos donde el 44,3\% califica la experiencia de buena y el 52,9\% de variable.

A quienes calificaron la experiencia de buena se les pidió que señalasen los beneficios que considerasen haber obtenido (Tabla IV).

Tabla IV. Si la experiencia ha sido buena, ¿qué beneficios obtuviste? (Señala tantas respuestas como sean necesarias)

\begin{tabular}{|l|l|l|}
\hline Opciones & $\mathbf{N . 3 1}$ & $\mathbf{\%}$ \\
\hline Fue una buena experiencia de aprendizaje & 28 & 90,3 \\
\hline Mejoré mis habilidades de escritura/presentación & 24 & 77,4 \\
\hline Me ayudó a completar mi conocimiento & 24 & 77,4 \\
\hline Me ayudó a entender el proceso de publicación científica & 20 & 64,5 \\
\hline $\begin{array}{l}\text { El reconocimiento académico conseguido fue beneficioso para el progreso } \\
\text { en mi carrera }\end{array}$ & 9 & 29,0 \\
\hline
\end{tabular}

En general, se percibe que las aportaciones de los revisores son valiosas y contribuyen al aprendizaje ayudando a mejorar los trabajos propios. Los participantes consideran mayoritariamente que la experiencia les ha permitido colmatar alguna laguna en su conocimiento. Un estudio anterior de Rodríguez-Bravo et al. (2017) apuntaba que en 2016 los españoles únicamente apoyaron con decisión la segunda respuesta de la tabla que hace referencia a la mejora de las habilidades para la escritura y la presentación. Con respecto al estudio que recoge datos globales de varios países se aprecia un apoyo superior al conocimiento obtenido del proceso de publicación (Jamali et al., 2020). Solo un tercio de los ECRs, tanto en España como a nivel general, considera haber obtenido algún reconocimiento por la tarea asumida de responder a los revisores. Tampoco en el trabajo mencionado previamente los ECRs señalaron que esta tarea fuese positiva para el avance de su carrera con la excepción de los franceses y malasios. 
Si la experiencia ha sido variable o mala se pide a los ECRs que señalen las principales razones (Tabla V). Se obtuvieron 41 respuestas una más de las que corresponden a quienes señalaron que la experiencia no había sido buena.

Tabla V. Si tu respuesta sobre la experiencia ha sido variable o mala ¿a qué se ha debido?

(Señala tantas respuestas como sean necesarias)

\begin{tabular}{|l|l|l|}
\hline Opciones & N. 41 & $\mathbf{\%}$ \\
\hline Los comentarios de los revisores fueron superficiales & 25 & 61,0 \\
\hline $\begin{array}{l}\text { Los comentarios de los revisores no demostraban conocimiento del } \\
\text { asunto del artículo }\end{array}$ & 23 & 56,1 \\
\hline El proceso de revisión fue demasiado largo & 20 & 48,8 \\
\hline Los revisores no fueron bien elegidos y no eran adecuados & 6 & 14,6 \\
\hline Los ECRs son tratados como novatos & 6 & 14,6 \\
\hline Los revisores no fueron receptivos a nuevas ideas & 8 & 19,5 \\
\hline No hubo oportunidad de responder al revisor tras recibir la revisión & 9 & 21,9 \\
\hline
\end{tabular}

Las tres razones más apoyadas son las que figuran en las primeras filas de la Tabla V. La superficialidad de los comentarios de los revisores es la principal causa elegida en España, mientras que la lentitud del proceso de revisión fue la respuesta más elegida a nivel global (Jamali et al., 2020). Como se ha apuntado, estudios previos (Rodríguez-Bravo et al., 2017; Rodríguez-Bravo y Nicholas, 2019) han indicado que uno de los principales motivos de descontento con el procedimiento reside en que los revisores elegidos no siempre son los idóneos y de ahí que sus comentarios sean superficiales y no demuestren conocimiento relevante del asunto del artículo.

La insatisfacción con el tiempo de respuesta de la revisión de sus artículos, contrasta curiosamente, con su opinión cuando son revisores de que algunas revistas no conceden suficiente tiempo para realizar un buen trabajo de revisión (Tabla VII). Se constata en este estudio nuevamente (Rodríguez-Bravo et al., 2017; RodríguezBravo y Nicholas, 2019) la queja de que los revisores no son receptivos a nuevas ideas, al igual que el malestar sentido de que no tratan a los ECRs con objetividad y que conduce, como se verá, a que los investigadores noveles sean partidarios del anonimato en el proceso de revisión.

Sí sorprende el relativamente elevado porcentaje de ECRs que manifiestan que su artículo se ha rechazado sin opción a réplica. ¿Quizá los editores se sienten más cómodos rechazando los trabajos de los investigadores junior que aquellos de los investigadores senior? Tres investigadores señalaron otras motivaciones y las argumentaron como sigue:

"He tenido experiencias en las que el revisor percibe el artículo como un ataque o incursión en "su campo" y destruye por completo el artículo. Por ejemplo, señala que no se ha citado "toda" su bibliografía (dejando clarísimo quién es el revisor) e intentando desprestigiar a los autores del artículo".

"Los revisores solicitaban datos que no era factible conseguir. Además, la falta de esos datos ya estaba justificada en uno de los apartados”. 
"En una ocasión uno de los revisores fue innecesariamente maleducado y agresivo en su lenguaje".

Estos comentarios son ilustrativos de las malas experiencias que acumulan los investigadores en el proceso de revisión, y no solo los noveles.

\subsection{La participación de los ECRs en el proceso de peer review como revisores, los beneficios conseguidos y su opinión sobre el proceso}

La primera cuestión de este apartado trataba de indagar sobre si los investigadores noveles habían actuado como revisores. De 111 respuestas el 43,2\% fueron afirmativas y el 56,8\% negativas. Se observa que algo menos de la mitad de quienes respondieron al cuestionario han actuado como revisores, siendo la situación a nivel global la inversa (Jamali et al., 2020).

Los 48 ECRs con experiencia respondieron a la siguiente pregunta que buscaba conocer cómo y por qué habían sido contactados la última vez.

Tabla VI. Piensa en la última vez que hiciste una revisión ¿cómo fuiste contactado?

\begin{tabular}{|l|l|l|}
\hline Opciones & $\mathbf{N . 4 8}$ & $\mathbf{\%}$ \\
\hline $\begin{array}{l}\text { Por medio de una invitación de la revista debido a mis publicaciones } \\
\text { previas en esa revista }\end{array}$ & 15 & 31,2 \\
\hline $\begin{array}{l}\text { Por medio de una invitación de la revista debido a mis publicaciones de } \\
\text { relevancia en otras revistas }\end{array}$ & 14 & 29,2 \\
\hline $\begin{array}{l}\text { Por medio de una invitación de mi mentor o del director del grupo de } \\
\text { investigación }\end{array}$ & 7 & 14,6 \\
\hline $\begin{array}{l}\text { Por medio de una invitación de la revista debido a mis contactos en el } \\
\text { comité editorial }\end{array}$ & 9 & 18,7 \\
\hline Porque soy miembro del comité editorial & 3 & 6,2 \\
\hline
\end{tabular}

Las razones que figuran en las dos primeras filas de la Tabla VI son las más comunes. La invitación procedió de la revista por la relevancia de las publicaciones previas del revisor, ya sea en la misma revista o en otras revistas. Cabe señalar también el papel desempeñado por los contactos en el comité editorial. Esta circunstancia parece más común en los campos de ciencias sociales y humanidades si se observan los datos globales del cuestionario (Jamali et al., 2020). La invitación del senior del grupo, puede ocultar la práctica cada vez más común de co-revisión o de revisión fantasma comentada previamente (McDowell et al., 2019) y de la que ya otros trabajos han ofrecido indicios (Nicholas et al., 2019). Formar parte del comité editorial no es frecuente en el estadio inicial de la carrera investigadora. Con todo, se constata que existen ciertas ocurrencias y esta circunstancia se ha confirmado respecto a ECRs experimentados de distintas ramas (Rodríguez-Bravo, et al., 2017).

Interesaba conocer también qué beneficios consideran los ECRs que les aporta la revisión por pares (Tabla VII). 
Tabla VII. ¿Qué has aprendido de tu labor como revisor? (Señala tantas respuestas como sean necesarias)

\begin{tabular}{|l|l|l|}
\hline Opciones & N. $\mathbf{4 7}$ & $\mathbf{\%}$ \\
\hline Cómo ser positivo y contribuir a la mejora del trabajo de otros & 39 & 83,0 \\
\hline $\begin{array}{l}\text { Identificar los errores de otros investigadores es una experiencia } \\
\text { provechosa }\end{array}$ & 33 & 70,2 \\
\hline Conocer lo que otros investigadores están haciendo & 28 & 59,6 \\
\hline Revisar consume mucho tiempo & 25 & 53,2 \\
\hline Resulta incómodo criticar/rechazar los manuscritos de los colegas & 16 & 34,0 \\
\hline La habilidad de los autores para la redacción es escasa & 13 & 27,7 \\
\hline $\begin{array}{l}\text { A los revisores no se les concede suficiente tiempo para realizar un } \\
\text { buen trabajo }\end{array}$ & 11 & 23,4 \\
\hline
\end{tabular}

Se aprecia en la Tabla VII que son cuatro las observaciones que registran el acuerdo de la mayoría. Revisando se aprende a ser constructivo para mejorar el manuscrito original. Contemporáneamente, se aprende de los errores de otros y se obtiene información sobre en qué trabajan nuestros peers. Se constata, asimismo, que la revisión implica una dedicación grande de tiempo y de ahí que se apunte que algunas revistas no ofrecen un plazo suficiente para realizar un buen trabajo. En este sentido, estudios anteriores señalaban que los ECRs hacían referencia, principalmente, a revistas Open Access cuando se lamentaban del poco tiempo que les dejaban algunas revistas para realizar el informe de revisión (Rodríguez-Bravo et al., 2017). Los ECRs se encuentran en una posición de debilidad y les incomoda revisar el trabajo de los investigadores senior, máxime si el proceso de revisión deja abiertas las identidades. La falta de calidad de algunos trabajos y su forma defectuosa también se puso de relieve en el estudio arriba citado donde algunos ECRs manifestaban que revisar trabajos pobres era una pérdida de tiempo.

En el cuestionario dos investigadores apuntaron otras razones:

"Cada vez que hago una revisión me pongo en el lugar del autor e intento comprender por qué y cómo hace su trabajo”. Esta respuesta reforzaría la primera opción de la tabla.

"Para artículos que usan datos es necesario disponer de éstos y del código para opinar con criterio". Esta respuesta se relaciona con la cada vez más sentida necesidad de que se publiquen los datos utilizados en las investigaciones para comprobaciones y a efectos de reproducibilidad.

\subsection{La valoración, preferencias y sugerencias de los ECRs sobre los modelos de revisión por pares}

A continuación, los jóvenes investigadores son preguntados por el sistema de revisión por pares que prefieren (Tabla VIII). 
Tabla VIII. ¿Qué tipo de revisión por pares prefieres como revisor? (elige una)

\begin{tabular}{|l|l|l|}
\hline Opciones & N. 106 & $\mathbf{\%}$ \\
\hline $\begin{array}{l}\text { Doble ciego (el revisor no conoce la identidad de los autores y } \\
\text { viceversa) }\end{array}$ & 52 & 49,1 \\
\hline $\begin{array}{l}\text { Triple ciego (el revisor no conoce la identidad de los autores y } \\
\text { viceversa. Además, los editores también desconocen las identidades de } \\
\text { autores y revisores) }\end{array}$ & 30 & 28,3 \\
\hline Identidades abiertas (el nombre de los revisores se publica) & 7 & 6,6 \\
\hline Informes abiertos (solo se hace público el contenido de la revisión) & 3 & 2,8 \\
\hline Ciego sencillo (el autor no sabe quiénes son los revisores) & 2 & 1,9 \\
\hline $\begin{array}{l}\text { Post-publicación (los artículos son revisados después de su } \\
\text { publicación) }\end{array}$ & 0 & 0,0 \\
\hline No tengo preferencia/No sé & 12 & 11,3 \\
\hline
\end{tabular}

Los ECRs se inclinan por la revisión anónima, siendo el doble ciego y el triple ciego las opciones preferidas con distancia respecto a las restantes modalidades presentadas. Recelan de sistemas más transparentes porque, como autores jóvenes creen que pueden verse perjudicados y como revisores sentirse costreñidos a manifestarse con cautela. En esta línea, el ciego sencillo, donde la identidad de los autores es conocida, la revisión post publicación abierta a la comunidad científica y la apertura de los informes son opciones que reciben un apoyo mínimo.

En el global de los resultados del cuestionario (Jamali et al., 2020) se observó que el sistema de doble ciego tenía más apoyo en el ámbito de las ciencias sociales que predomina en las respuestas al cuestionario en España y que podría ser responsable, en parte, del notable apoyo que recibe esta opción. A juzgar por los datos obtenidos en el estudio citado, los investigadores de ciencias de la vida serían algo más proclives a sistemas con identidades abiertas y a informes abiertos, mientras que en ciencias de la salud parece más común la utilización del modelo de ciego sencillo.

En este sentido, cabe apuntar que se sabe que los sistemas de revisión varían en función de las disciplinas y los investigadores no siempre conocen más de uno que es el que tienden a respaldar. Así, Rodríguez-Bravo y Nicholas (2019) recogen que un científico español del área de informática, con una asombrosa experiencia como revisor, abogaba por el ciego sencillo argumentando que era el sistema usual en su área y que presentaba la ventaja de poder comprobar que los autores estaban realizando una aportación distinta a las publicadas en trabajos previos. Este es uno de los puntos fuertes señalados por los muy escasos investigadores que, según los datos de Rodríguez-Bravo et al. (2017) basados en las entrevistas previas, apoyaron a nivel global el modelo open peer review junto con que el revisor se ve obligado a ser más profesional y educado en sus comentarios al resultar expuesto al juicio de sus pares Es notable, asimismo, el porcentaje de investigadores que no manifiesta preferencia lo que concuerda con un número importante de científicos que no han sido revisores, algunos de los cuales no han participado en absoluto en el proceso de revisión. Se pregunta a continuación por las razones de las preferencias señaladas (Tabla IX). 
Tabla IX. ¿Cuál es la razón principal de tu elección? (Elige un único motivo)

\begin{tabular}{|l|l|l|}
\hline Opciones & N. 101 & \% \\
\hline $\begin{array}{l}\text { El anonimato es crucial para una revisión honesta y neutral. Puede } \\
\text { impedir la parcialidad, el comportamiento inadecuado y el abuso del } \\
\text { revisor }\end{array}$ & 88 & 87,1 \\
\hline La transparencia potencia la rendición de cuentas y la minuciosidad & 8 & 7,9 \\
\hline La transparencia inhibe la expresión de críticas y valoraciones negativas & 1 & 1,0 \\
\hline
\end{tabular}

El apoyo al anonimato en la revisión es incuestionable (Tabla IX), si bien superior en España que en el conjunto de los países que han respondido al cuestionario. A nivel global (Jamali et al., 2020) se aprecia que hay investigadores que apoyan sistemas transparentes, fundamentalmente porque promueven la rendición de cuentas y la minuciosidad en la revisión, como ya hemos apuntado. El hecho de que puedan filtrar en exceso las críticas recibe menos apoyo.

Cuatro participantes ofrecieron otras razones. Algunas que denotan desconocimiento: "No sé”; "No he revisado". Indecisión: "Hay ventajas y desventajas en todos los métodos" o que apoyan la primera opción de la tabla: "En mi caso, al ser investigadora muy novel (en el primer año de redacción de la tesis), considero que el anonimato puede servir para que se tome más en serio mi propuesta”. Esta última respuesta que se refiere a la posición del investigador como autor que espera pacientemente la revisión de su manuscrito ilustraría la preocupación porque no se tome en serio el trabajo de los ECRs que se ha ya comentado en el apartado anterior (Tabla V), donde se observó que el 14,6\% de los españoles encuestados se lamentaban de ser tratados como novatos.

Pese a que los ECRs se hallan medianamente satisfechos con el proceso de peer review, a la pregunta: ¿¿Se puede mejorar el sistema de revisión por pares para los ECRs? el 90,7\% responde afirmativamente. La siguiente pregunta sobre cómo mejorar el proceso de arbitraje se formuló utilizando una escala de Likert: Mucho (5), Bastante (4), Algo/Poco (3), Muy poco (2), Nada (1), No sé (0). La respondieron 96 investigadores y la Tabla $\mathrm{X}$ refleja los valores medios.

Tabla X. ¿Hasta qué punto consideras que las siguientes modificaciones pueden mejorar la revisión por pares para los ECRs?

\begin{tabular}{|l|l|}
\hline Opciones & Valor \\
\hline Unos comentarios más constructivos para la mejora de un artículo & 4,53 \\
\hline Los revisores debieran estar más abiertos a las ideas nuevas & 4,27 \\
\hline Una evaluación más rigurosa del mérito y de la novedad de un artículo & 4,11 \\
\hline $\begin{array}{l}\text { La revisión por pares en cascada debe ser adoptada. En ella la revisión } \\
\text { obtenida se traslada de una revista que ha rechazado el artículo a la siguiente } \\
\text { revista a la que se envía el artículo }\end{array}$ & 3,66 \\
\hline La revisión post-publicación sería una mejora & 3,30 \\
\hline
\end{tabular}

En la Tabla X se comprueba el gran respaldo que obtienen todas las opciones. No sorprende el apoyo recabado por las tres afirmaciones primeras dado que ya 
habíamos constatado la queja de que los revisores no siempre hacían una revisión suficientemente completa y que con frecuencia eran refractarios a ideas nuevas. En la Tabla V se apreciaba que casi el $20 \%$ de los participantes españoles considera que los revisores no siempre son sensibles a la innovación. Dado el considerable tiempo de espera por el informe de revisión, excesivo en opinión de los ECRs, como se deja ver en la Tabla $\mathrm{V}$, y que se duplica o triplica si un artículo resulta finalmente rechazado y se tiene que comenzar el proceso con otra revista, no extraña que se apoyen nuevas prácticas del tipo de la revisión en cascada. En los datos del cuestionario global al que nos hemos referido (Jamali et al., 2020) se aprecia más apoyo a la revisión en cascada entre los investigadores de ciencias de la salud y ciencias de la vida. Sí parece menos congruente el apoyo a la revisión post publicación, dado que en la Tabla VIII se puede constatar que ningún ECR español apoyó este modelo de revisión, probablemente por desconocimiento.

Para finalizar con las preguntas sobre el proceso de revisión, se deseaba conocer si los ECRs españoles creen que es apropiado que sean los editores quienes gestionen el peer review. Dos tercios (66,7\%) de 105 investigadores ha respondido afirmativamente. El apoyo a los editores ha sido constatado también en otros estudios previos (Rodríguez-Bravo, et al., 2017; Jamali et al., 2020).

En la última pregunta (Tabla XI) se solicitan otras opciones a quienes respondieron negativamente a la cuestión anterior.

Tabla XI. Si tu respuesta es negativa, ¿quién debiera hacerlo? (Elige una respuesta)

\begin{tabular}{|l|l|l|}
\hline Opciones & N. 35 & $\mathbf{\%}$ \\
\hline Un servicio independiente de revisión por pares & 15 & 42,9 \\
\hline $\begin{array}{l}\text { Las comunidades de investigadores por medio de sus repositorios } \\
\text { temáticos (por ejemplo BioRXiv) }\end{array}$ & 12 & 34,3 \\
\hline Las sociedades científicas & 4 & 11,4 \\
\hline Las bibliotecas y/o las redes sociales & 2 & 5,7 \\
\hline Las instituciones educativas & 2 & 5,7 \\
\hline
\end{tabular}

Las alternativas con mayor aceptación serían las que aparecen en las primeras filas de la Tabla XI: un servicio independiente, las comunidades de investigadores por medio de los repositorios temáticos y las sociedades científicas. Con todo, no parece que los investigadores noveles valoren otras alternativas seriamente como han constatado estudios recientes de Tennant (2018) y de Nicholas et al. (2018).

\section{Discusión y conclusiones}

El trabajo de Nicholas et al. (2019), cuando evalúa la evolución experimentada por los ECRs entre 2016 y 2018 a partir de la información obtenida de las entrevistas realizadas en la primera fase del Proyecto Harbingers, constata que los principales cambios se habían producido en el terreno de la colaboración, la persecución del impacto social y la utilización de las redes sociales. Otros trabajos (Nicholas, et al., 
2020a; Rodríguez-Bravo y Nicholas, 2019) también inciden en la creciente importancia que los investigadores noveles dan a la colaboración. Los ECRs son conscientes de su valor para el avance de sus carreras así como para la transferencia de conocimiento. La colaboración tiene consecuencias en la mejora de calidad de la investigación y también en el incremento de la coautoría, con las implicaciones que hemos señalado en este estudio.

En otros ámbitos -y pese a sus creencias de millenials- los ECRs actúan convencionalmente suprimiendo sus tendencias a la apertura y la transparencia en pos de la seguridad. En los estudios mencionados, al igual que en el de Jamali et al., (2020), se ha constatado que el objetivo principal de los ECRs es publicar muchos artículos en una posición prominente en la lista de autores o ser autor de correspondencia. Por este motivo la autoría es un área de gran controversia donde los investigadores noveles se lamentan de que se concedan coautorías injustificadas o se ordene el ranking de autores sin reflejar el trabajo que se ha invertido en el artículo. Sin embargo, en el caso español, Rodríguez-Bravo y Nicholas (2019) constataron que cuando publicaban material de su tesis doctoral eran primeros autores y que cuando eran ya ECRs senior podían pasar a ocupar la posición de autor de correspondencia. El mismo trabajo confirma que los ECRs estaban más satisfechos en 2018 que en 2016 porque habían entendido las reglas del juego y se habían vuelto más pragmáticos. Se aprueba que el orden pueda alterarse para beneficiar a algún investigador que tenga que concursar para obtener una nueva posición en la carrera científica.

Los ECRs se vuelcan en publicar en revistas revisadas por pares y con alto factor de impacto. Expresan su total convencimiento de que para su futuro es imprescindible (Nicholas et al., 2020b). Por tanto, pese a que en la dinámica actual y en el entorno colaborativo la revista tradicional parece estar dejando de adecuarse a las necesidades de la ciencia (Stern y O'Shea, 2019), los imperativos personales, que persiguen obtener crédito, influyen más en las decisiones de donde publicar que cualquier otra consideración y las revistas reconocidas, de "marca", son la norma y lo seguirán siendo mientras gobiernen los procesos de evaluación de la investigación (Herman et al., 2020).

Los investigadores noveles son conscientes que en el éxito de su carrera como investigadores el arbitraje juega un papel crucial. De ahí que en la elección de la revista donde envían el manuscrito la calidad y rapidez del peer review sea también un factor a considerar (Nicholas et al, 2017; Rodríguez-Bravo et al., 2017). Los jóvenes investigadores creen que existe vinculación entre las revistas bien posicionadas y la calidad de su arbitraje. En Rodríguez-Bravo y Nicholas (2019) una investigadora española así lo afirmaba. Argumentaba que en ocasiones se enviaba un manuscrito a una revista top solo para que la revisión obtenida les ayudara a mejorarlo para otra revista menos reputada.

Sorprendentemente el proceso de peer review no genera tantas críticas como habríamos anticipado dado el obstáculo que supone en el camino de la publicación. Aunque califican de variable su experiencia los ECRs se muestran relativamente positivos. Prefieren el sistema de doble ciego porque les permite permanecer en el anonimato como autores y también como revisores dado que temen ser perjudicados 
por su débil posición en la academia, precaución confirmada en los estudios de Segado-Boj; Martín-Quevedo y Prieto-Gutiérrez (2018) y Rodríguez-Bravo et al. (2017). Los españoles se muestran especialmente conservadores por el entorno competitivo en el que tratan de mantenerse a flote (Rodríguez-Bravo y Nicholas, 2019). El interés por sistemas de identidades abiertas es limitado, si bien quienes tienen experiencia en esos modelos de arbitraje destacan sus fortalezas.

Como trabajos basados en las entrevistas del Proyecto Harbingers confirman (Rodríguez-Bravo et al.,2017; Rodríguez-Bravo y Nicholas, 2019), los investigadores noveles mayoritariamente se sienten reconocidos porque se cuente con ellos para una tarea tan crítica en el proceso de publicación. Piensan también que es justo que todos los investigadores contribuyan a un proceso que dota de calidad de la ciencia. Con todo, algunos estudios detectan además la petición de formación para acometer la tarea revisora y la solicitud de reconocimiento por ella (RodríguezBravo, et al., 2017; Rodríguez-Bravo y Nicholas, 2019; Young, Mitterboeck, 2020; Warne, 2016). El reconocimiento, redundaría en un mejor y más rápido trabajo de revisión. La plataforma Publons ha sido un paso adelante porque facilita a los ECRs demostrar su actividad de revisión en cualquier concurso o promoción laboral.

Opinan que los editores debieran estar atentos y evaluar el trabajo de los revisores a quienes encomiendan esta responsabilidad. Como señalaba una investigadora española "habría que revisar a los revisores a partir del feedback de los autores" (Rodríguez-Bravo y Nicholas, 2019). Pese a las debilidades detectadas, los ECRs se inclinan porque sigan siendo los editores quienes organicen la revisión por pares y parece que no valoran seriamente otras alternativas como han puesto de relieve previamente otros estudios (Nicholas et al., 2018; Tennant, 2018).

Otros estudios centrados en el peer review (Casnici, et al., 2016) indican que los ECRs son más voluntariosos y concienzudos como revisores que los investigadores consolidados: reciben más solicitudes, las aceptan con mayor frecuencia y son más rápidos enviando sus respuestas. No obstante, los ECRs más experimentados aluden al abuso de los editores que les tienen en cuenta para revisar por la calidad de su trabajo y, sin embargo, no les ofrecen un puesto en el comité editorial (Jamali et al., 2020). Los resultados de la encuesta gestionada por McDowell et al. (2019) refleja que tres cuartas partes de los ECRs que contestaron han revisado papers con sus mentores y opinan que es una actividad beneficiosa (95\%) y ética (73\%) que contribuye a su formación. Sin embargo, la mitad han realizado también esa revisión de manera solitaria y anónima (ghostwriting) por delegación de sus mentores pese a considerar esa forma de comportamiento no ética (81\%) y opinar que las revistas tienen que identificar a los co-revisores (82\%). Nicholas et al. (2019) también ponen de manifiesto la actuación de los investigadores junior por delegación de sus mentores. El proceso de peer review se beneficiaría de cambios en las políticas de las revistas y en las prácticas de los grupos de investigación que incentivasen la revisión guiada por investigadores expertos (Young \& Mitterboeck, 2020) y pusiera trabas a la revisión fantasma de ECRs y al abuso de los investigadores al mando.

Para finalizar queremos subrayar que autoría y peer review son procesos muy vinculados y de ahí nuestro interés en tratarlos conjuntamente. Los resultados obtenidos de la encuesta realizada a ECRs españoles refuerzan aquellos hallados en 
otros estudios que indagan, así mismo, sobre las percepciones de los investigadores noveles (Jamali et al., 2020; Nicholas et al., 2017, 2019, 2020a, 2020b; RodríguezBravo et al., 2017; Rodríguez-Bravo y Nicholas, 2019; Young y Mitterboeck, 2020) y, por tanto, refuerzan las conclusiones que exponemos a continuación.

La principal es que los investigadores noveles son un colectivo experimentado y multitarea que contribuye enormemente tanto a la elaboración y publicación de resultados científicos como a su filtro por medio de la revisión. En este estudio se constata la generalizada ausencia de políticas de autoría en España. Los ECRs, sin embargo, desearían que las reglas que rigen la posición de los autores fueran fijas y que, en todo caso, la decisión se tomase de manera consensuada. Mayoritariamente han presentado artículos para su revisión y han contestado a los comentarios de los revisores, si bien en menor porcentaje que en el conjunto de los países analizados en el trabajo de Jamali et al. (2020). Asimismo, muchos han sido revisores y aparentemente consideran que el sistema funciona todo lo bien que sería esperable habida cuenta de las tensiones asociadas con la evaluación. Las críticas se centran en el excesivo tiempo que tienen que esperar por las revisiones (si bien como revisores entienden que el proceso es complejo y no les gusta que les den plazos cortos). Asimismo, se quejan de que los árbitros no son siempre los apropiados para juzgar un artículo y que no son receptivos a nuevas ideas. Con todo, respecto al arbitraje, los ECRs se muestran conservadores, prefieren mantener el anonimato, y aunque quieren pequeñas mejoras se escabullen de cambios radicales.

\section{Referencias bibliográficas}

Baldwin M. (2018). Scientific autonomy, public accountability, and the rise of "Peer Review" in the Cold War United States. Isis, 109, 538-558. doi: 10.1086/700070

Casnici, N.; Grimaldo, F.; Gilbert, N. y Squazzoni, F. (2016). Attitudes of referees in a multidisciplinary journal: an empirical analysis. Journal of the Association for Information Science and Technology, 68 (7), 1763-1771. https://doi.org/10.1002/asi.23665

Herman, E.; Akeroyd, J.; Bequet, G.; Nicholas, D. y Watkinson, A. (2020). The changed and changing- landscape of serials publishing: review of the literature on emerging models. Learned Publishing (en prensa). https://doi.org/10.1002/leap.1288

Inside eLife (2018). Early-career researchers: views on peer review. https://elifesciences.org/ inside-elife/982053f4/early-career-researchers-views-on-peer-review [Consulta: 11/05/2020]

Jamali, H. R.; Nicholas, D.; Watkinson, A.; Abrizah, A.; Rodríguez-Bravo, B.; BoukacemZeghmouri, C.; Xu, J.; Polezhaeva, T.; Herman, E. y Swigon, M. (2020). Early career researchers and their autorship and peer review beliefs and practices: An international study. Learned Publishing, 33 (2), 142-152. https://doi.org/10.1002/leap.1283

McDowell, G. S. (2018). Early-career researchers and their involvement in peer review. https://asapbio.org/mcdowell-ecrs [Consulta: 11/05/2020]

McDowell, G. S.; Knutsen, J. D.; Graham, J. M.; Oelker, S. K. y Lijek, R. S. (2019). Coreviewing and ghostwriting by early-career researchers in the peer review of manuscripts. eLife, 8. doi: 10.7554/eLife.48425

Merga, M. K.; Mason, S. y Morris, J. (2018). Early career experiences of navigating journal article publication: lessons learned using an autoethnographic approach. Learned Publishing, 31 (4), 381-389. https://doi.org/10.1002/leap.1192 
Nicholas, D.; Watkinson, A.; Boukacem-Zeghmouri, C.; Rodríguez-Bravo, B.; Xu, J.; Abrizah, A.; Swigon, M. y Herman, E. (2019). So, are early career researchers the harbingers of change?, Learned Publishing, 32 (3), 237-247. https://doi.org/10.1002/leap.1232

Nicholas, D.; Watkinson, A.; Jamali, H. R.; Herman, E.; Tenopir, C.; Volentine, R.; Allard, S. y Levine, K. (2015). "Peer review: still King in the digital age”. Learned Publishing, 28 (1), 15-21. doi:10.1087/20150104

Nicholas, D.; Rodríguez-Bravo, B.; Watkinson, A.; Boukacem-Zeghmouri, C.; Herman, E.; Xu, J.; Abrizah, A. y Swigon, M. (2017). Early career researchers and their publishing and authorship practices. Learned Publishing, 30 (3), 205-217. doi.10.1003/leap.1102

Nicholas, D.; Watkinson, A.; Abrizah, A.; Boukacem-Zeghmouri, C.; Xu, J.; Rodríguez Bravo, B.; Świgoń, M. y Herman, E. (2018). What publishers can take away from the latest early career researcher research. Learned Publishing, 31 (3), 249-253. doi:10.1002/leap.1165

Nicholas, D.; Watkinson, A.; Abrizah, A.; Rodríguez-Bravo, B.; Boukacem-Zeghmouri, C.; Xu, J.; Świgon, M. y Herman, E.(2020a). Does the scholarly communication system satisfy the beliefs and aspirations of new researchers? Summarizing the Harbingers research. Learned Publishing, 33 (2), 132-141. https://doi.org/10.1002/leap.1284

Nicholas, D.; Jamali, Hamid R.; Herman, E.; Watkinson, A.; Abrizah, A.; Rodríguez-Bravo, B.; Boukacem-Zeghmouri, C.; Xu, J.; Swigon, M. y Polezhaeva, T. (2020b). A global questionnaire survey of the scholarly communication attitudes and behaviours of early career researchers, Learned Publishing, 33 (en prensa), https://doi.org/10.1002/leap.1286

Patterson, M. y Schekman, R. (2018). How early-career researchers are shaping eLife. eLife, 7, e36263. doi: 10.7554/eLife.36263.

Rodríguez- Bravo, B.; Nicholas, D. (2019). Reputación y comunicación científica. El caso de los investigadores españoles en el inicio de su carrera. El Profesional de la información, 28 (2), e280203.

Rodríguez-Bravo, B.; Nicholas, D.; Herman, E.; Boukacem-Zeghmouri, C.; Watkinson, A.; Xu, J.; Abrizah, A. y Swigon, M. (2017). Peer review: the experience and views of early career researchers. Learned Publishing, 30 (4), 269-277. https://onlinelibrary.wiley.com/ doi/epdf/10.1002/leap.111

Segado-Boj, F.; Martín-Quevedo, J. y Prieto-Gutiérrez, J.-J.. (2018). Attitudes toward open access, open peer review, and altmetrics among contributors to Spanish scholarly journals. Journal of Scholarly Publishing, 50 (1), 48-70. doi: 10.3138/jsp.50.1.08

Stern, B. M.; O’Shea, E. K. (2019). A proposal for the future of scientific publishing in the life science. PloS Biology, 17 (2). https://doi.org/10.1371/journal.pbio.3000116

Tennant, J. P. (2017). A multi-disciplinary perspective on emergent and future innovations in peer review” [version 3; peer review: 2 approved] F1000Research, 6 (1151). doi: 10.12688/f1000research.12037.3.

Tennant, J. P. (2018). The state of the art in peer review. FEMS Microbiology Letters, 365, (19), fny204. https://doi.org/10.1093/femsle/fny204

Warne, V. (2016). Rewarding reviewers - Sense or sensibility? A Wiley study explained. Learned Publishing, 29 (1), 41-50. https://doi.org/10.1002/leap.1002

Weber, M. (2018). The effects of listing authors in alphabetical order: a review of the empirical evidence. Research Evaluation, 27, 238-245.

Young, R. G.; Mitterboeck, T. F. (2020). Perspectives for early-career researchers on plagiarism and scientific integrity. FACETS: a multidisciplinary open access science journal, 5, 17-25. https://doi.org/10.1139/facets-2019-0031. 